\title{
Mode coupling analysis of the dynamical structure factor in supercooled liquid $\mathrm{Na}_{0.5} \mathrm{Li}_{0.5} \mathrm{PO}_{3}$
}

\author{
B. Rufflé ${ }^{\mathrm{a}, *}$, S. Beaufils ${ }^{\text {a }}$, B. Toudic ${ }^{\text {a }}$, C. Ecolivet ${ }^{\text {a }}$, A. Le Sauze ${ }^{\text {b }}$, \\ R. Marchand ${ }^{\mathrm{b}}$ \\ ${ }^{a}$ Groupe Matière Condensée et Matériaux, UMR CNRS 6626, Université Rennes I, Campus de Beaulieu, 35042 Rennes cedex, France \\ ${ }^{\mathrm{b}}$ Groupe Verres et Céramiques, Université Rennes I, Campus de Beaulieu, 35042 Rennes cedex, France
}

\begin{abstract}
The dynamical structure factor of supercooled $\mathrm{Na}_{0.5} \mathrm{Li}_{0.5} \mathrm{PO}_{3}\left(T_{\mathrm{g}}=515 \mathrm{~K}, T_{\mathrm{m}}=749 \mathrm{~K}\right)$ has been obtained by time-offlight neutron scattering between room temperature and $773 \mathrm{~K}$, just above the melting temperature. The temperature and wave number dependences of the measured spectra in the whole supercooled liquid range are shown to be well described by the solutions of a schematic mode coupling theory model. In particular, the crossover between vibrational excitations and fast relaxation processes above the susceptibility minimum is reproduced by the calculated spectra. This analysis leads then to the identification of a crossover temperature around $620 \mathrm{~K}$ in this non-fragile glass-forming liquid. (c) 1998 Elsevier Science B.V. All rights reserved.
\end{abstract}

\section{Introduction}

All glass-forming liquids exhibit between the melting temperature $T_{\mathrm{m}}$ and the calorimetric glass transition temperature $T_{\mathrm{g}}$ a strong increase in their transport coefficients like viscosity. However the temperature dependence is a specific feature of the system. A classification of supercooled liquids can be made by plotting the logarithm of the viscosity against the temperature reduced scale $T_{\mathrm{g}} / T$ [1]. All data are then found between two limiting cases, denoted as strong and fragile liquids. Strong liquids such as $\mathrm{SiO}_{2}$ are characterized by an Arrhenius law and usually form tridimensional covalent networks. On the other hand, fragile liquids

\footnotetext{
${ }^{*}$ Corresponding author. Present address: Hahn-Meitner-Institut, Abt. NI, Glienicker str. 100, D-14109 Berlin, Germany. E-mail: ruffle@hmi.de.
}

(molten salt mixtures or Van der Waals liquids) follow a highly non-Arrhenius behavior between a low viscosity regime at high temperature and a high viscosity regime around $T_{\mathrm{g}}$ separated by a narrow crossover temperature range.

Recently, this crossover temperature region has been the subject of increasing interest [2] in connection with the recent development of a new theoretical approach. The mode coupling theory (MCT) of the liquid-glass transition [3] provides a consistent description of the dynamics in supercooled liquids. An avoided ergodic non-ergodic transition is predicted to occur above $T_{\mathrm{g}}$ in the weak supercooled liquid regime revealing specific features around the crossover temperature $T_{\mathrm{c}}$ for all variables that couple to density fluctuations. A two step decay of the correlation functions should be observed around $T_{\mathrm{c}}$ leading to the identification of a short time dynamics in the picoseconds ( $\beta$-process) and a long time temperature 
sensitive dynamics ( $\alpha$-process). Asymptotic developments of the mode coupling equations close to the singularity provide handy formulas to test the validity of the MCT.

Recent experimental data obtained mostly on fragile glass-forming liquids strongly support the MCT at least concerning the temperature range above $T_{\mathrm{c}}$ that is usually found around $20 \%$ above $T_{\mathrm{g}}$ [4-6]. However, when going towards least fragile supercooled liquids, discrepancies arise between data and mode coupling predictions [7,8]. Indeed, low frequency vibrational excitations that form the so-called boson peak are known to increase in intensity with decrease of fragility and thus strongly influence the dynamics in the frequency range that is exactly concerned by some scaling laws predicted by the MCT [9]. Furthermore, the range of validity of these predictions remains unknown for such complicated liquids. Another way to test the validity of the MCT in less fragile liquids is then to resolve the mode coupling equations within the frame of a schematic model and compare the complete solution with the experimental data $[10,11]$. This approach provides the advantage to take into account the specific microscopic dynamics of the system under study that cannot be described with asymptotic formulas.

In this paper, we report an analysis of the dynamical structure factor of $\mathrm{Na}_{0.5} \mathrm{Li}_{0.5} \mathrm{PO}_{3}$ obtained by coherent neutron scattering measurements in the whole supercooled liquid range. The temperature and wave number dependences of the scattered spectra are described by the solutions of a schematic mode coupling theory model. After a short Section 2 giving some details on the system under study and on the experimental setup, Section 3 describes precisely the schematic mode coupling theory model used to fit the data. The results are presented in Section 4 and discussed in Section 5 whereas conclusions appear in Section 6.

\section{Experimental details}

$\mathrm{Na}_{0.5} \mathrm{Li}_{0.5} \mathrm{PO}_{3}$ is a mixed alkali phosphate glassforming liquid that can be easily supercooled down to the glass transition temperature $T_{\mathrm{g}}$ without any sign of crystallization. It forms long twist- ed chains of covalent bonded $\mathrm{PO}_{4}$ tetrahedra of about 100 units. The structure is then close to the one of a polymer where both cations $\mathrm{Li}^{+}$and $\mathrm{Na}^{+}$play the role of side groups. Although $\mathrm{Na}_{0.5} \mathrm{Li}_{0.5} \mathrm{PO}_{3}$ is an oxide glass, its characteristic temperatures remain in a convenient domain, i.e. $T_{\mathrm{g}}=515 \mathrm{~K}$ and $T_{\mathrm{m}}=749 \mathrm{~K}$. Neutron scattering experiment has been carried out on the multichopper time-of-flight (TOF) spectrometer MIBEMOL at the Laboratoire Léon Brillouin ${ }^{1}$ in the temperature range $300-773 \mathrm{~K}$ with an energy resolution of $87 \mu \mathrm{eV}$. More details concerning the experimental setup and the synthesis of the sample used for this experiment can be found elsewhere [12].

\section{Schematic mode coupling theory model}

The mode coupling theory of the liquid-glass transition assumes that the dynamics of the supercooled liquid is mainly governed by the density fluctuations. In this approach, the normalized wave number dependent correlation functions of density fluctuations are calculated from the following set of closed equations of motion with the initial conditions $\phi_{q}(0)=1, \dot{\phi}_{q}(0)=0$ :

$$
\begin{gathered}
\ddot{\phi}_{q}(t)+v_{q} \dot{\phi}_{q}(t)+\Omega_{q}^{2} \phi_{q}(t)+\Omega_{q}^{2} \\
\int_{0}^{t} m_{q}\left(t-t^{\prime}\right) \dot{\phi}_{q}\left(t^{\prime}\right) \mathrm{d} t^{\prime}=0, \\
m_{q}(t)=\mathscr{F}_{q}\left[V, \phi_{q}(t)\right],
\end{gathered}
$$

where $\Omega_{q}$ and $v_{q}$ characterize the microscopic dynamics of the liquid. The non-trivial part of the MCT is the evaluation of the memory function $m_{q}(t)$ in Eq. (2) in order to describe the cage effect [3]. Indeed, $m_{q}(t)$ is developed as a polynomial $\mathscr{F}_{q}$ of the correlation functions $\phi_{q}(t)$ where the positive coefficients of the polynomial $(V)$ are completely determined by the static structure factor $S(q)$. These coupling parameters increase smoothly with decreasing temperature leading to a kinematic instability where the supercooled liquid structure

\footnotetext{
${ }^{1}$ Laboratoire Léon Brillouin is a laboratoire commun CEACNRS.
} 
becomes frozen. For more details concerning the various predictions of the mode coupling theory, the reader is referred to the review [3].

The system under study is intermediate between strong and fragile glass forming liquid so that the dynamics present some specific features that prevent a simple mode coupling analysis of the data with leading order asymptotic results [12]. On the other hand, the mode coupling functional $\mathscr{F}_{q}$ is not known for $\mathrm{Na}_{0.5} \mathrm{Li}_{0.5} \mathrm{PO}_{3}$. Thus, for testing the MCT in this non-fragile supercooled liquid, we have used a two-component schematic mode coupling theory model as a basic description of the dynamics in $\mathrm{Na}_{0.5} \mathrm{Li}_{0.5} \mathrm{PO}_{3}$. The first component is the well studied $\mathrm{F}_{12}$ model [3] dealing with one correlator $\phi(t)$. It uses a linear and a quadratic term in the memory function with two coupling parameters $V_{1}$ and $V_{2}, m(t)=V_{1} \phi(t)+V_{2} \phi^{2}(t)$. $\phi(t)$ is meant to represent all the density fluctuations in the supercooled liquid $\mathrm{Na}_{0.5} \mathrm{Li}_{0.5} \mathrm{PO}_{3}$. It drives the critical behavior from the weak coupling liquid state to the strong coupling glass state leading to the ergodic non-ergodic transition at $T_{\mathrm{c}}$. A second correlator $\phi_{\mathrm{s}}(Q, t)$ coupled to the first one is added in order to describe the dynamics of the correlation function probed by the experiment and will be $Q$-dependent in this study. The memory function is then specified by the single coupling parameter $V_{\mathrm{s}}(Q), m_{\mathrm{s}}(Q, t)=V_{\mathrm{s}}(Q) \phi(t) \phi_{\mathrm{s}}(Q, t)$.

This model, first proposed by Sjögren [13], has been also used to describe the tagged particles dynamics of a liquid and more recently depolarized light scattering susceptibility spectra measured in Glycerol [10]. It should be pointed out that the first correlator $\phi(t)$ that drives the critical behavior influences the dynamics of $\phi_{\mathrm{s}}(t)$ but the reverse is not true. It ensures a critical transition occuring for both correlators at the same time for well known sets of coupling parameters $\left(V_{1}, V_{2}\right)$.

\section{Results}

In order to achieve the fits presented below, the four parameters $\Omega, v, \Omega_{\mathrm{s}}$ and $v_{\mathrm{s}}$ characterizing the high frequency dynamics of both correlators $\phi$ and $\phi_{\mathrm{s}}$ have been kept constant so that temperature and wave number dependences of the calcu- lated dynamical structure factor are completely included in the smooth temperature drift of the coupling parameters $V_{1}, V_{2}, V_{\mathrm{s}}$ and the modulation of $V_{\mathrm{s}}$ with $Q$. The schematic mode coupling theory model has been numerically resolved in the time domain using an algorithm similar to the one described in Ref. [14]. After a Fourier transform of $\phi_{\mathrm{s}}(Q, t)$, the calculated dynamical structure factor was obtained by multiplying $\phi_{\mathrm{s}}^{\prime \prime}(Q, \omega)$ with the static structure factor $S(Q), S(Q, \omega)=S(Q) \phi_{\mathrm{s}}^{\prime \prime}$ $(Q, \omega)$. The convoluted spectra, $S^{\text {conv }}(Q, \omega, T)=$ $S(Q, \omega, T) \otimes R(Q, \omega)$, where $R(Q, \omega)$ is the resolution function of the spectrometer, was then compared to the complete experimental dynamical structure factor including the ill-resolved part of the spectra that appears as a narrow quasielastic component.

Fig. 1 shows some calculated susceptibility spectra $\chi^{\prime \prime}(Q, \omega, T)=S(Q, \omega, T) / n(\omega, T)$, where $n(\omega, T)$ is the Bose factor, compared to the experimental susceptibility spectra in a log-log plot for selected temperatures at $Q=1.8 \AA^{-}$. In this plot, experimental data below $0.1 \mathrm{meV}$ influenced by

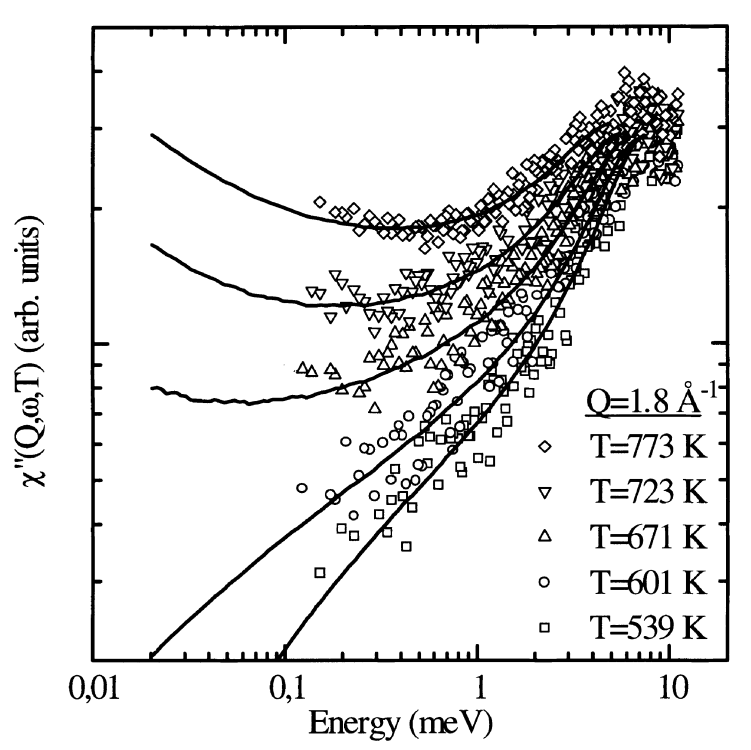

Fig. 1. Experimental coherent neutron scattering susceptibility spectra (symbols) $\chi^{\prime \prime}(Q, \omega, T)=S(Q, \omega, T) / n(\omega, T)$ at selected temperatures between $T_{\mathrm{g}}$ and $T_{\mathrm{m}}$ for $Q=1.8 \AA^{-1}$ in $\mathrm{Na}_{0.5} \mathrm{Li}_{0.5} \mathrm{PO}_{3}$ compared to the susceptibility calculated with the schematic mode coupling theory model (lines). 


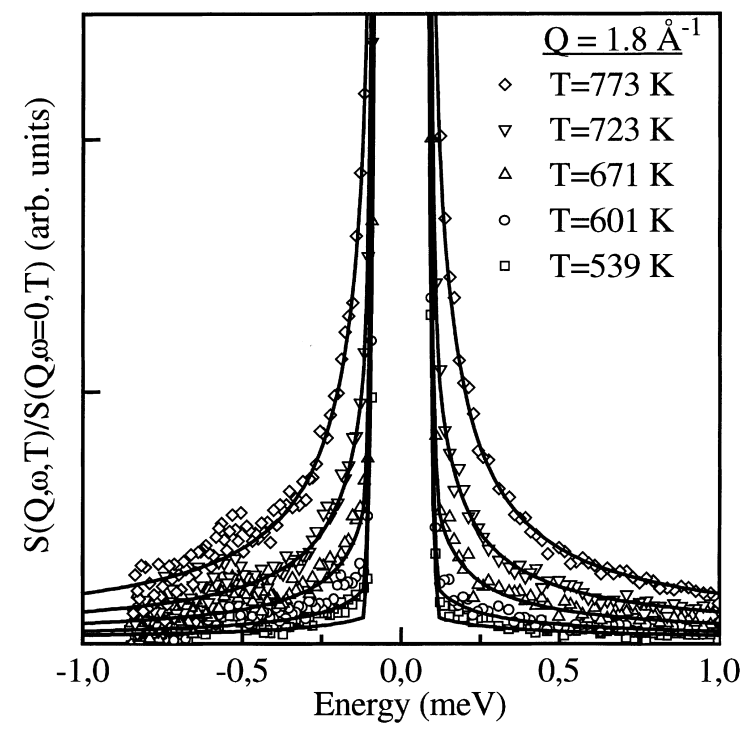

Fig. 2. Temperature dependence of the experimental dynamical structure factor (symbols) for $Q=1.8 \AA^{-1}$ in $\mathrm{Na}_{0.5} \mathrm{Li}_{0.5} \mathrm{PO}_{3}$ compared to the dynamical structure factor computed from the schematic mode coupling theory model and convoluted with the resolution function of MIBEMOL (lines).

the resolution function have been removed. In Fig. 2, the temperature evolution of the low energy quasielastic part of the experimental dynamical structure factor at the same wave number is plotted and compared to the convoluted calculated spectra $S^{\text {conv }}(Q, \omega, T)$ showing a very good agreement at all the temperatures. At this point, it can be stated that the schematic mode coupling theory model used in this study describes very well the temperature dependence of the spectra when the coupling parameters $V_{1}$ and $V_{2}$ are simply changed with temperature.

Furthermore, using $Q$-independent coupling parameters $V_{1}$ and $V_{2}$, this schematic model can also fit experimental spectra obtained at different wave numbers when the third coupling parameters $V_{\mathrm{s}}$ is varied. Indeed, Fig. 3 shows the low frequency part of the experimental dynamical structure factor for selected wave numbers at $T=773 \mathrm{~K} \mathrm{com-}$ pared to the convoluted solutions of the schematic mode coupling theory model where only $V_{\mathrm{s}}$ is varied. It appears clearly that the narrowing of the quasielastic scattering due to the structural relaxation around the first maximum of the static struc-

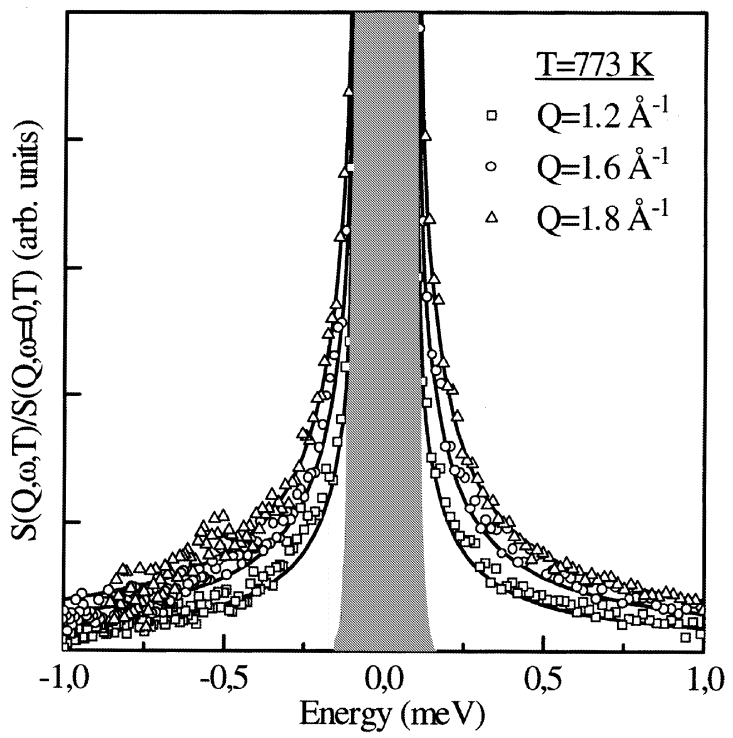

Fig. 3. $Q$-dependence of the experimental dynamical structure factor (symbols) at $T=773 \mathrm{~K}$ in $\mathrm{Na}_{0.5} \mathrm{Li}_{0.5} \mathrm{PO}_{3}$ compared to the dynamical structure factor computed from the schematic mode coupling theory model and convoluted with the resolution function of MIBEMOL (lines). The coupling parameters $V_{1}$ and $V_{2}$ are the same for all the spectra whereas $V_{\mathrm{s}}$ is $Q$-dependent. The shaded area is the resolution function.

ture factor at $Q=1.2 \AA^{-1}$ is very well reproduced by the schematic model.

\section{Discussion}

These results suggest that the dynamics measured in the non-fragile glass-forming liquid matches the physical picture given by the MCT as for fragile liquids. Indeed, as can be seen in Fig. 1, the crossover region between fast processes and vibrational motions exhibited by the experimental data around $1-5 \mathrm{meV}$ is completely reproduced by the solutions of the schematic model. The transition from a sublinear frequency dependence of the susceptibility at low energy towards a linear frequency dependence at higher energy revealing the boson peak is clearly demonstrated at low temperature in the calculated spectra. The shallow susceptibility minimum that appears above $671 \mathrm{~K}$ in the experimental data between the Boson peak and the incoming structural 
relaxation is also correctly reproduced at all the temperatures. At the lowest energy, the complex shape of the structural relaxation, its temperature and wave number dependences are also well described as clearly seen in Figs. 2 and 3.

One of the most important output of the analysis is obviously the trajectory of $\left(V_{1}, V_{2}\right)$ in the coupling parameters plane. As shown in Fig. 4, the coupling parameters $\left(V_{1}, V_{2}\right)$ used to achieve the fits cross the bifurcation line of the $F_{12}$ model between 601 and $637 \mathrm{~K}$ in this system thus around $1.2 T_{\mathrm{g}}$. It is striking to notice that the trajectory seems to be very similar to the one obtained recently in Glycerol, another non-fragile glass-former, with the same schematic model but on depolarized light scattering data [10]. The inset of Fig. 4 shows the wave number dependence of the third mode coupling parameter $V_{\mathrm{s}}$ that has been used to fit the spectra at $T=773 \mathrm{~K}$. The $Q$-dependence exhibits a marked peak at $1.2 \AA^{-1}$ corresponding to the location of the first maximum of the static structure factor in this system. In the frames of this schematic MCT model, it leads to a narrowing of the $\alpha$-process also exhibited by the experimental data as clearly shown in Fig. 3. At higher $Q$ values, $V_{\mathrm{s}}$ decreases rapidly according to the Debye-Waller factor and, in spite of a very

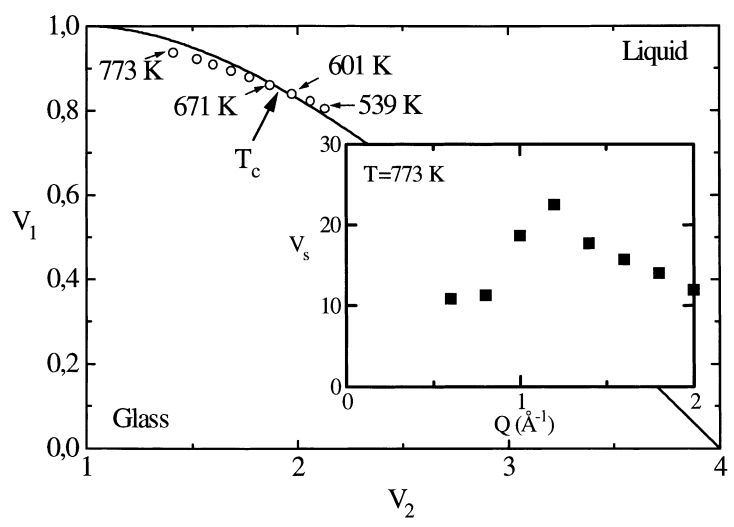

Fig. 4. Trajectory in the coupling parameters plane of $\left(V_{1}, V_{2}\right)$ used to describe the data. The critical line is crossed between 601 and $637 \mathrm{~K}$, i.e. at $1.2 T_{\mathrm{g}}$ in this system. The inset shows the wave number dependence of the third coupling parameter $V_{\mathrm{s}}$ of the schematic model. A marked peak around the first maximum of the static structure factor at $1.2 \AA^{-1}$ is clearly seen whereas at higher $Q$ values, $V_{\mathrm{s}}$ decreases rapidly. simple schematic model used, similarly to the $Q$ dependence of the critical form factor at the critical point computed recently with the complete mode coupling equations in a hard-spheres system [15].

\section{Conclusions}

We have shown that the dynamics probed by a coherent neutron scattering experiment in the nonfragile glass-forming liquid $\mathrm{Na}_{0.5} \mathrm{Li}_{0.5} \mathrm{PO}_{3}$ could be completely described by the solutions of a schematic mode coupling theory model dealing with two correlators. One for the density fluctuations that governs the critical dynamics and monitors the slowing down of the structural relaxation and a second one that represents the correlation function probed by the experiment and thus that can be compared to the experimental data. The smooth drift with temperature of the coupling parameters $\left(V_{1}, V_{2}\right)$ occuring in the first component leads to a good description of the temperature evolution of the susceptibility spectra as well as the quasielastic shape. This analysis leads to a crossover temperature $T_{\mathrm{c}}$ located between 601 and 637 $\mathrm{K}$ in this non-fragile system, thus around $1.2 T_{\mathrm{g}}$ as for most fragile liquids investigated so far. Furthermore, the $Q$-dependence of the scattered spectra is also reproduced by the schematic model simply by the modulation of the third coupling parameter $V_{\mathrm{s}}$ with $Q$.

\section{References}

[1] C.A. Angell, J. Phys. Chem. Solids 49 (1988) 863.

[2] See, e.g., special issue, K.L. Ngai, E. Riande, G.B. Wright (Eds.), J. Non-Cryst. Solids 172-174 (1994).

[3] For a review, see W. Götze, L. Sjögren, Rep. Prog. Phys. 55 (1992) 241.

[4] W. Knaak, F. Mezei, B. Farago, Europhys. Lett. 7 (1988) 529.

[5] G. Li, W.M. Du, X.K. Chen, H.Z. Cummins, N.J. Tao, Phys. Rev. A 45 (1992) 3867.

[6] J. Wuttke, M. Kiebel, E. Bartsch, F. Fujara, W. Petry, H. Sillescu, Z. Phys. B 91 (1993) 357.

[7] J. Wuttke, J. Hernandez, G. Li, G. Coddens, H.Z. Cummins, F. Fujara, W. Petry, S. Sillescu, Phys. Rev. Lett. 72 (1994) 3052. 
[8] M.J. Lebon, C. Dreyfus, G. Li, A. Aouadi, H.Z. Cummins, R.M. Pick, Phys. Rev. E 51 (1995) 4537.

[9] A.P. Sokolov, E. Rössler, A. Kisliuk, D. Quitmann, Phys. Rev. Lett. 71 (1993) 2062.

[10] T. Franosch, W. Götze, M.R. Mayr, A.P. Singh, Phys. Rev. E 55 (1997) 3183.

[11] V. Krakoviack, C. Alba-Simionesco, M. Krauzman, J. Chem. Phys. 107 (1997) 3417.
[12] B. Rufflé, J. Etrillard, B. Toudic, C. Ecolivet, J.P. Ambroise, G. Coddens, E. Guéguen, R. Marchand, Phys. Rev. B 56 (1997) 11546.

[13] L. Sjögren, Phys. Rev. A 33 (1986) 1254.

[14] M. Fuchs, W. Götze, I. Hofacker, A. Latz, J. Phys.: Condense. Mat. 3 (1991) 5047.

[15] T. Franosch, M. Fuchs, W. Götze, M.R. Mayr, A.P. Singh, Phys. Rev. E 55 (1997) 7153. 